Notas Clínicas

\title{
Reacción de llamarada en el cáncer de mama avanzado
}

\author{
A. S. Rubiales, M. L. del Valle, L. Gómez, E. Uña, S. Hernansanz, L. A. Flores
}

\section{Resumen}

- Propósito: La reacción de llamarada se manifiesta de inicio como un aumento aparente de la actividad tumoral inducido por el propio tratamiento. No es raro que se presente como un incremento del metabolismo de las lesiones tumorales, principalmente óseas, sin embargo es infrecuente que aparezca junto con otros problemas como dolor o alteración metabólica.

- Material y métodos: Presentamos dos pacientes con cáncer de mama avanzado que desarrollaron esta reacción de llamarada inducida en un caso por el tratamiento hormonal (tamoxifeno) y en el otro por quimioterapia.

- Resultados: Ambas pacientes presentaron de inicio un aumento del dolor que sugería progresión de la enfermedad asociado a una elevación de los marcadores. Más tarde se evidenció una clara respuesta del tumor. Destaca el manuscrito en que la propia paciente describe cómo vive los síntomas que se le presentan.

- Conclusiones: La reacción de llamarada puede manifestarse como un aparente empeoramiento transitorio de la enfermedad al que sigue la respuesta tumoral.

Palabras clave:

Reacción de llamarada. Cáncer de mama avanzado.

Oncología, 2005; 28 (1):45-49

Servicio de Oncología

Hospital Clínico Universitario

Valladolid 


\section{Summary}

- Purpose: Flare reaction presents initially as a treatment-induced apparent rise of tumor activity. It can manifest as an increase of metabolism in the tumor lesions, most frequently bone metastases. The presentation involving other manifestations as pain or metabolic disorders is infrequent.

- Material and methods: We present two patients with advanced breast cancer that developed a flare reaction induced by hormone therapy (tamoxifen) in one case, and by chemotherapy in the other.

- Results: Both patients suffered initially an increase in pain intensity with a parallel elevation of tumor markers. Later was evidenced a clear response. We underline the manuscript of the second patient where she describes her own feelings and symptoms during the flare.

- Conclusions: Flare reaction can manifest as an apparent transient worsening of the disease, followed up by an evident tumor response.

Key words: Flare reaction. Advanced breast cancer.

\section{Introducción}

La reacción de llamarada (flare) es un fenómeno poco frecuente en que la aparición de una respuesta importante inmediata se manifiesta de inicio como un aumento aparente de la actividad tumoral inducido por el propio tratamiento'. Desde el punto de vista clínico el paciente refiere un empeoramiento brusco de los síntomas con un aumento transitorio del dolor óseo $^{2}$. A este cuadro se pueden asociar alteraciones metabólicas como la hipercalcemia ${ }^{3}$. Además, en los estudios que muestran la actividad de las lesiones tumorales (gammagrafía, PET) puede apreciarse un aumento transitorio de la actividad ${ }^{4}$, que algunos autores ponen en relación con la activación de los osteoblastos ${ }^{5}$. Esta reacción de llamarada se ha descrito en el cáncer de mama avanzado debida a diferentes tipos de tratamiento hormonal (antiestrógenos, análogos $\mathrm{LH}-\mathrm{RH}$, inhibidores de la aromatasa, progestágenos) ${ }^{6}$, así como a quimioterapia ${ }^{7}$. No hay cifras realmente fiables sobre su incidencia de ya que por una parte los datos suelen proceder de series retrospectivas $y$, por otra, el criterio diagnóstico no es uniforme ya que es más fácil encontrarlo con una monitorización bioquímica estrecha que con el mero seguimiento clínico ${ }^{3,5}$.

Por la muy escasa frecuencia de los casos con manifestaciones clínicas, especialmente los inducidos por quimioterapia, presentamos la evolución de dos pacientes que desarrollaron cuadros compatibles con reacción de llamarada, en la primera inducido por tratamiento hormonal (tamoxifeno ${ }^{8}$ ) y en la segunda por quimioterapia que incluía antraciclinas, taxanos y trastuzumab.

\section{Pacientes}

\section{Paciente 1}

Mujer de 80 años que refería la presencia de una tumoración en la mama izquierda desde hacía dieciséis años de la que no había querido consultar por miedo al diagnóstico y a los posibles tratamientos. Aunque refería que al principio el crecimiento había sido lento e indolente, en los dos últimos años había progresado hasta el punto llegar a autoamputar la mama. Había acudido finalmente a la Consulta animada por sus familiares tras presentar dolor dorsal y disnea progresiva, más evidentes en las semanas previas. Presentaba en ese momento adenopatías axilares y supraclaviculares ipsilaterales. En la radiografía de tórax se encontraron signos de derrame pleural y una probable linfangitis carcinomatosa (Fig. 1). La gammagrafía ósea mostraba captaciones múltiples. El diagnóstico clínico se confirmó mediante punción de un nódulo que sobresalía sobre el lecho mamario; la citología fue compatible con carcinoma ductal infiltrante. La paciente comenzó tratamiento con tamoxifeno. Dos días después presentó de manera repentina un empeoramiento importante de los dolores dorsales por lo que fue preciso aumentar la analgesia. En las dos semanas siguientes la propia paciente fue retirando los analgésicos hasta llegar a suspenderlos. Tras una elevación transitoria, tanto el CA153 como la fosfatasa alcalina fueron disminuyendo hasta alcanzar niveles normales. Al cabo de seis meses se había obtenido una respuesta completa de las adenopatías si bien persistía un pequeño resto en el lecho mamario; la gammagrafía mostraba una cierta atenuación de las 

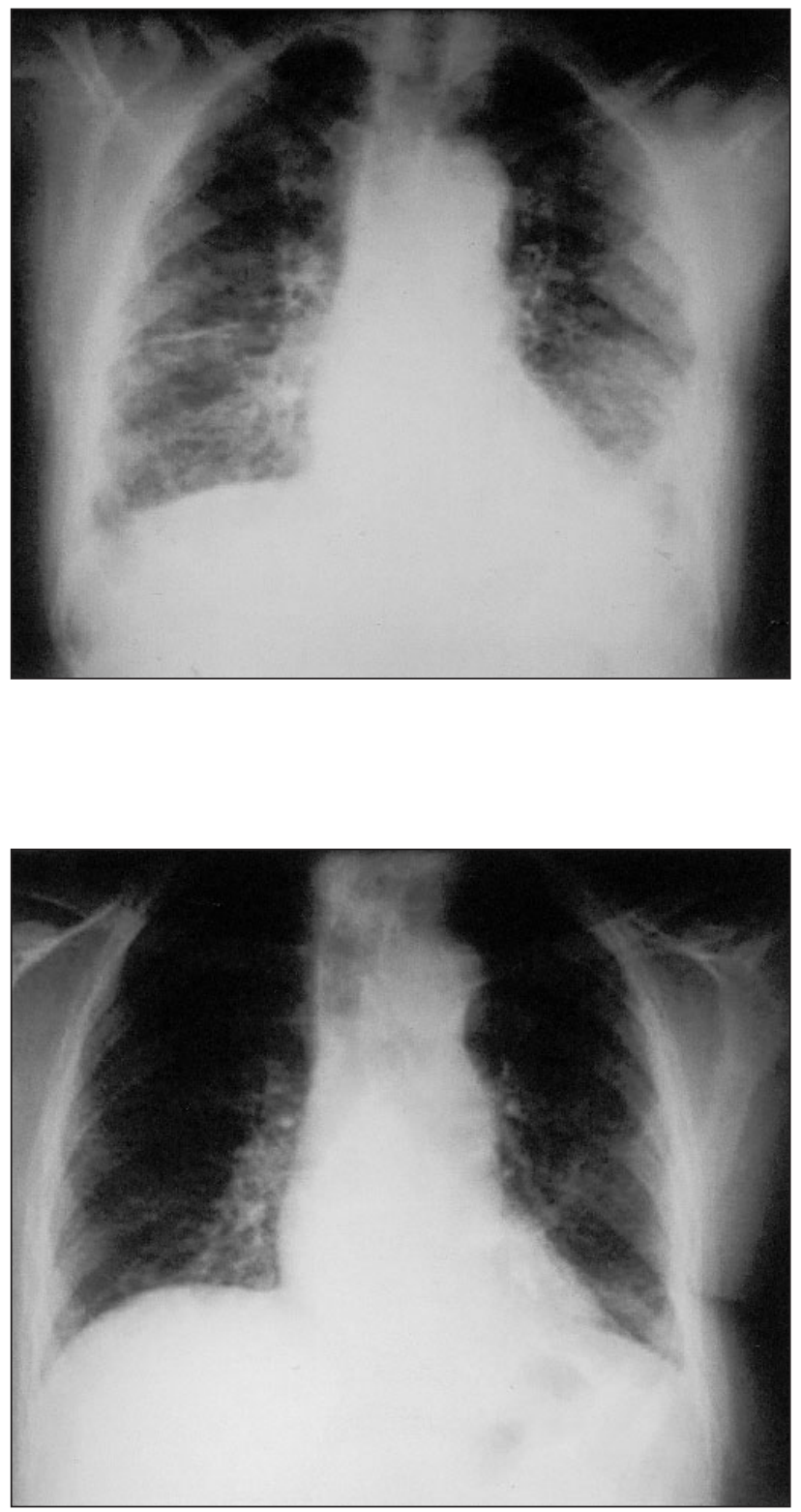

Fig. 1. Radiografía de tórax de la paciente 1 antes y después del tratamiento con tamoxifeno.

zonas hipercaptantes y se apreciaba una clara mejoría de la afectación pulmonar (Fig. 1). La paciente mantuvo la respuesta durante 22 meses. Tras la progresión recibió varias líneas de tratamiento hormonal. Falleció al cabo de 56 meses del diagnóstico.

\section{Paciente 2}

En el año 2000, a los 47 años de edad, se le practicó una mastectomía por un carcinoma ductal infiltrante de mama estadio IIIB (T4a N1). Los receptores hormo-

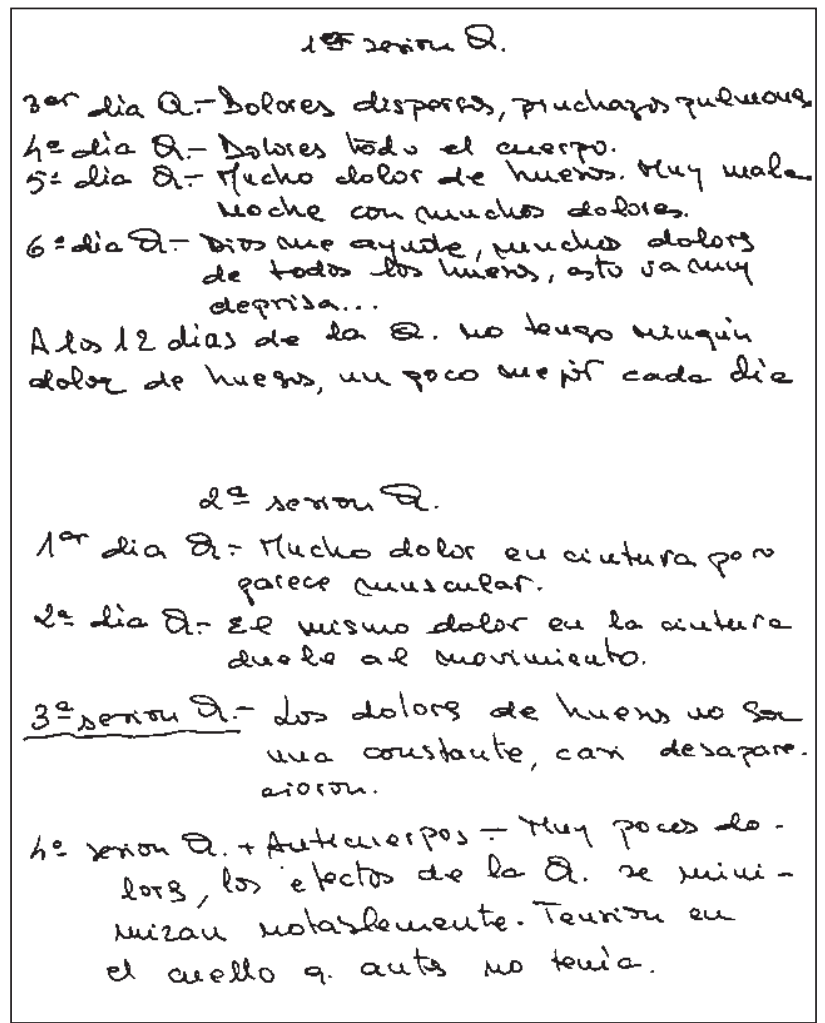

Fig. 2. Descripción de la paciente 2 de los síntomas durante la reacción de llamarada.

nales eran positivos, al igual que el Herceptest (+++). Recibió tratamiento complementario con quimioterapia (CMF), radioterapia y tamoxifeno. En Mayo de 2003 se detectó una recidiva ósea cervical que ocasionaba compromiso medular. Fue tratada con cirugía y radioterapia local. Posteriormente comenzó tratamiento con letrozol. En Noviembre de 2003 se confirmó una progresión tumoral ósea y ganglionar (supraclavicular). Comenzó entonces tratamiento citostático paliativo con epirrubicina, docetaxel y trastuzumab. En las semanas siguientes presentó un empeoramiento sintomático importante pero transitorio; la evolución de estos síntomas se describe en unas anotaciones de la propia paciente que luego nos facilitó (Fig. 2). De manera simultánea se encontró una elevación del CA153 y de la fosfatasa alcalina que posteriormente se fue reduciendo hasta normalizarse (Gráfica 1). Desde el punto de vista clínico, la captación en la gammagrafía ósea no sufrió cambios relevantes pero la adenopatía supraclavicular alcanzó una respuesta completa. La duración de la respuesta fue de diez meses, tras los cuales presentó progresión ósea y hepática. Se encuentra recibiendo una nueva línea de quimioterapia con aparente estabilización de la enfermedad. 


\section{A. S. Rubiales y cols.}

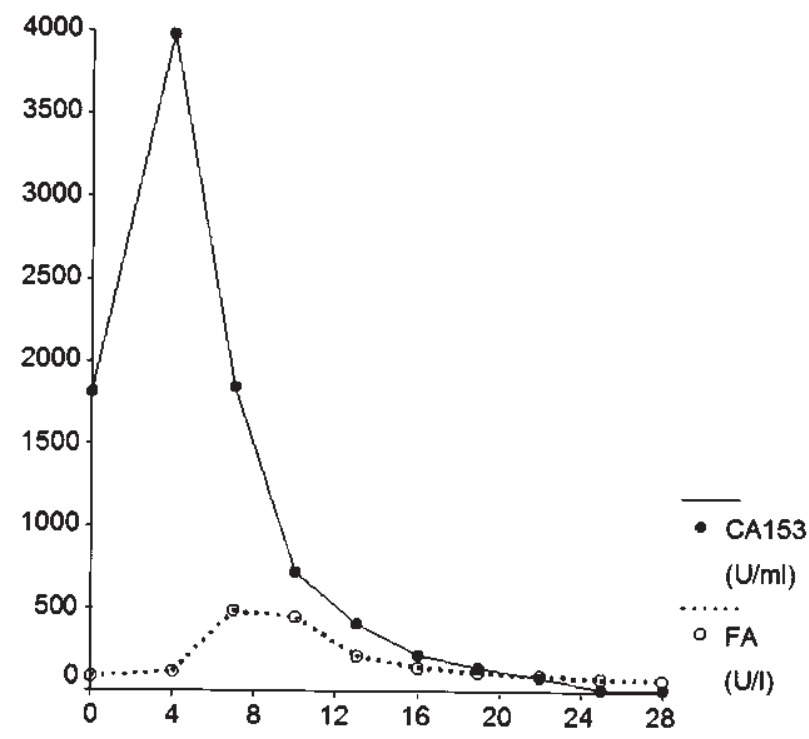

semanas desde el inicio del tratamiento

Gráfica 1. Evolución de los niveles séricos de CA153 y fosfatasa alcalina (FA) desde el inicio del tratamiento con quimioterapia en la paciente 1.

\section{Comentario}

La reacción de llamarada es un fenómeno clínico poco frecuente. En nuestra experiencia, la aparición de una reacción de llamarada con manifestaciones clínicas evidentes sucede en menos del $1 \%$ de las enfermas con cáncer de mama avanzado que recibe tratamiento sistémico, hormonal o con quimioterapia. Tanto la escasa frecuencia como la perplejidad que produce un fenómeno en que el empeoramiento clínico puede ser el primer aviso de una respuesta evidente pueden hacer que en más de una ocasión no se diagnostique de manera adecuada?.

El tamoxifeno es un antiestrógeno parcial que consigue respuestas objetivas en cerca de una de cada tres pacientes con cáncer de mama avanzado. Esta proporción aumenta cuando se seleccionan tumores con receptores hormonales positivos. Y es el tamoxiferno el fármaco con el que se han descrito más casos de reacción de llamarada en cáncer de mama avanzado. El hecho de que su mecanismo de acción esté mediado por un receptor específico puede favorecer que haya tumores con una sensibilidad extraordinaria a este tratamiento hormonal. En la Paciente 1 no hay datos sobre la situación de los receptores hormonales. No se insistió en conocer este dato ya que previsiblemente no iba a modificar la actitud terapéutica una vez que la propia paciente había descartado cualquier opción de tratamiento con quimioterapia. Se describe que el cáncer de mama avanzado con afectación extravisceral tiene un comportamiento más indolente y mejor pronóstico ${ }^{10}$. El comportamiento del cáncer en esta paciente, con un antecedente mantenido de más de quince años, hace pensar en un tumor indolente que sólo en los últimos meses o años se había mostrado más agresivo. El perfil de la paciente, salvo por la linfangitis pulmonar, marcaba una alta probabilidad de respuesta a tratamiento hormonal. $Y$, efectivamente, se encontró una respuesta duradera al tratamiento hormonal en partes blandas (pared torácica) y ganglios pero también en la probable linfangitis pulmonar, una complicación para la que se suele recomendar un tratamiento más agresivo. Esta respuesta vino precedida por el empeoramiento temprano, a las 48 horas del inicio del tratamiento, que aparece como manifestación clínica de la reacción de llamarada. Se trata de un fenómeno paradójico en que la exacerbación de los síntomas es el primer aviso de una respuesta clínica ulterior, habitualmente clara y mantenida.

Los casos que se han publicado de reacción de llamarada por quimioterapia en cáncer de mama avanzado son aún más escasos ${ }^{1,7}$. Y la mayor parte se diagnosticaron únicamente por los cambios en la intensidad de captación de la gammagrafía, sin referencia a las manifestaciones clínicas del paciente. En relación con la Paciente 2, no hemos encontrado casos previos (Medline, Cochrane, IME) de reacción de llamarada inducida por docetaxel, epirrubicina o trastuzumab.

Aunque sólo se presentan los datos de la Paciente 2 (Gráfica 1), en las dos enfermas se encontró una elevación paradójica al inicio del tratamiento del CA153 y de la fosfatasa alcalina paralelo al empeoramiento del dolor ${ }^{11}$. Es interesante leer de puño y letra de la paciente cómo en paralelo a alteración bioquímica ella vive un empeoramiento de su enfermedad. Y eso supone un sufrimiento doble, por una parte el dolor óseo y por otra la angustia y el miedo anticipatorio a una progresión rápida e irreversible del cáncer. Es interesante mantener cierta actitud de sospecha, también sobre este fenómeno de la reacción de llamarada, algo que facilita y adelanta el diagnóstico de muchas complicaciones.
Correspondencia:

Dr. A. S. Rubiales

Oncología Médica

Hospital Clínico Universitario

C/ Ramón y Cajal, 3

E-47005 Valladolid

E-mail: asrubiales@hotmail.com 


\section{Bibliografía}

1. Mortimer JE, Dehdashti F, Siegel BA, Trinkaus K, Katzenellenbogen JA, Welch MJ. Metabolic flare: indicator of hormone responsiveness in advanced breast cancer. J Clin Oncol 2001; 19: 2797-803.

2. Plotkin D, Lechner JJ, Jung WE, Rosen PJ. Tamoxifen flare in advanced breast cancer. JAMA 1978; 240: 26446.

3. Koizumi M, Matsumoto S, Takahashi S, Yamashita T, Ogata $\mathrm{E}$. Bone metabolic markers in the evaluation of bone scan flare phenomenon in bone metastases of breast cancer. Clin Nucl Med 1999; 24: 15-20.

4. Vogel CL, Schoenfelder J, Shemano I, Hayes DF, Gams RA. Worsening bone scan in the evaluation of antitumor response during hormonal therapy of breast cancer. J Clin Oncol 1995; 13: 1123-8.

5. Coleman RE, Whitaker KB, Moss DW, Mashiter G, Fogelman I, Rubens RD. Biochemical prediction of response of bone metastases to treatment. $\mathrm{Br}$ J Cancer 1988; 58: 205-10.
6. Clarysse A. Hormone-induced tumor flare. Eur J Cancer Clin Oncol 1985; 21 : 545-7.

7. Schneider JA, Divgi CR, Scott AM, et al. Flare on bone scintigraphy following Taxol chemotherapy for metastatic breast cancer. J Nucl Med 1994; 35: 1748-52.

8. Plotkin D, Lechner JJ, Jung WE, Rosen PJ. Tamoxifen flare in advanced breast cancer. JAMA 1978; 240: 26446.

9. Coleman RE, Mashiter G, Whitaker KB, Moss DW, Rubens RD, Fogelman I. Bone scan flare predicts successful systemic therapy for bone metastases. J Nucl Med 1988; 29: 1354-9.

10. Osborne CK. Tamoxifen in the treatment of breast cancer. N Engl J Med 1998; 339:1609-18.

11. Berruti A, Osella G, Raucci CA, Roncari A, Dogliotti L. Transient increase in total serum alkaline phosphatase predicts radiological response to systemic therapy in breast cancer patients with osteolytic and mixed bone metastases. Oncology 1993; 50: 218-21. 